\title{
Soluble ICAM-1 serum levels in patients with intermediate uveitis
}

\author{
Anne-Marie Klok, Leny Luyendijk, Michel J W Zaal, Aniki Rothova, Aize Kijlstra
}

\section{Department Ophthalmo- Immunology, Netherlands Ophthalmic Research Institute, Amsterdam A-M Klok L Luyendijk A Kijlstra}

Department of Ophthalmology, Free University, Amsterdam A-M Klok M J W Zaal

Department of Ophthalmology, University of Amsterdam, Amsterdam A-M Klok A Kijlstra

Department of Ophthalmology, University of Utrecht, Utrecht

A Rothova

Correspondence to: Anne-Marie Klok, Department

Ophthalmo-Immunology, Netherlands Ophthalmic Research Institute, Amsterdam, PO Box 12141, 1100 AC Amsterdam, Netherlands.

Accepted for publication 12 January 1999

\begin{abstract}
Aim-To investigate whether serum levels of soluble intercellular adhesion molecule 1 (sICAM-1) can serve as a marker of the presence of systemic disease in intermediate uveitis.

Methods-In a multicentre study sICAM-1 serum levels were measured in 61 patients with idiopathic intermediate uveitis, controls included 56 uveitis patients with a systemic disease (26 sarcoid associated uveitis and 30 HLA-B27 positive acute anterior uveitis), 58 uveitis patients without systemic disease (30 toxoplasma chorioretinitis and 28 Fuchs' hetrochromic cyclitis), and 21 normal controls. The clinical records of the patients with intermediate uveitis were analysed for disease characteristics at the time of blood sampling and for a relation with the development of a systemic disease after a mean follow up of 4.5 years.

Results-Increased serum levels of sICAM-1 were found in 34 out of 61 patients with intermediate uveitis and were significantly different when compared with toxoplasmosis, Fuchs' cyclitis, and healthy controls $(p<0.001)$. Elevated sICAM-1 levels were also found in 18 out of 26 patients with sarcoid uveitis and in 11 out of 30 patients with HLA-B27 associated anterior uveitis. Raised SICAM-1 levels in the intermediate uveitis group were significantly associated with active ocular disease $(p<0.01)$ and the presence of vitreous exudates $(p<0.05)$. Increased levels of sICAM-1 correlated with interleukin 8 levels (IL-8) (tested in a previous study in the same group of intermediate uveitis patients) in patients with active systemic involvement. Follow up of the patients showed that an established or suspected systemic disease was found more often in the 21 intermediate uveitis patients with increased SICAM-1 and IL-8 levels compared with the other 40 patients with intermediate uveitis $(\mathbf{p}<0.01)$.

Conclusions-The measurement of both sICAM-1 and IL-8 can be used as a marker for ocular disease activity and for a predisposition of developing an associated systemic disease in intermediate uveitis patients.

(Br f Ophthalmol 1999;83:847-851)
\end{abstract}

Intermediate uveitis accounts for approximately $8-16 \%$ of uveitis cases. ${ }^{1-3}$ The diagnosis of intermediate uveitis is made when ocular inflammation predominantly involves the vitre- ous and peripheral retina. ${ }^{14}$ The disease tends to mostly affect young adults although it can also be diagnosed later in life. The aetiology of intermediate uveitis is still unknown. The association of intermediate uveitis with systemic diseases such as multiple sclerosis and sarcoidosis suggests that at least some cases of this heterogeneous ocular disorder are autoimmune in nature..$^{5-7}$ In cases of idiopathic intermediate uveitis it is possible that an underlying systemic disease may not yet be obvious.

In an earlier study we found increased levels of interleukin 8 (IL-8) in patients with active intermediate uveitis, a phenomenon which suggests a systemic involvement. ${ }^{8}$ Other immune mediators such as intercellular adhesion molecule 1 (ICAM-1) were also reported to be upregulated in retinal inflammatory diseases. ${ }^{9}$ ICAM-1 is an adhesion molecule belonging to the immunoglobulin superfamily that is expressed on cells of multiple lineage at sites of inflammation $^{10-14}$ It is important for leucocyte adhesion, transendothelial migration, and cellcell interactions in immune responses. ${ }^{15}$ It has been suggested that ICAM-1 is an early marker of immune activation and response. A circulating soluble form of ICAM-1 (sICAM-1) has been detected in human serum. ${ }^{16}{ }^{17}$ High levels of sICAM-1 have been reported in inflammatory and autoimmune diseases such as rheumatoid arthritis, spondylarthropathies, sarcoidosis, and multiple sclerosis. ${ }^{18-23}$ Furthermore, it has been suggested that high levels of sICAM-1 correlate well with disease activity in multiple sclerosis, ${ }^{22}{ }^{23}$ sarcoidosis, ${ }^{20}{ }^{21}$ and also in patients with retinal vasculitis. ${ }^{24}$

Whether sICAM-1 can also be useful as an indicator for the presence of systemic disease in intraocular inflammation is not yet clear and was the main objective of this study.

\section{Patients and methods}

Sixty one patients with intermediate uveitis who visited the uveitis clinics of the Academic Hospital of the Free University of Amsterdam and the Academic Hospital of Utrecht in the period between 1981 and 1996 were included in this study (this group of intermediate uveitis patients was also included in our previous study for IL- $\left.8^{8}\right)$. All patients underwent a complete examination for uveitis, ${ }^{25}$ a complete ophthalmological examination was performed in all cases, and their personal and family histories were reviewed. The patients were especially asked for arthralgias, skin rash, gastrointestinal symptoms, and neurological symptoms. Blood samples for uveitis screening (erythrocyte sedimentation rate, $\mathrm{C}$ reactive protein, red and white blood cell counts, 
Table 1 Elevated sICAM-1 levels (>300 $\mathrm{ng} / \mathrm{ml}$ ) in serum of uveitis patients

\begin{tabular}{lrrrr}
\hline Diagnosis & No/total & $\%$ & $\begin{array}{l}\text { Median } \\
(\text { ng/ml })\end{array}$ & $\begin{array}{l}\text { Range } \\
(\text { ng/ml })\end{array}$ \\
\hline Intermediate uveitis & $34 / 61$ & 56 & 337 & $181-684$ \\
Sarcoidosis & $18 / 26$ & 69 & 504 & $146-1585$ \\
HLA-B27 AAU & $11 / 30$ & 37 & 298 & $175-472$ \\
Toxoplasmosis & $7 / 30$ & 23 & 265 & $145-439$ \\
Fuchs' cyclitis & $1 / 28$ & 4 & 238 & $177-316$ \\
Controls & $0 / 21$ & - & 203 & $98-287$
\end{tabular}

AAU $=$ acute anterior uveitis.

${ }^{\star}$ sICAM-1 levels in intermediate uveitis were significantly different compared with toxoplasmosis, Fuchs' cyclitis, and controls $(\mathrm{p}<0.001$, Mann-Whitney $\mathrm{U}$ test $)$.

angiotensin converting enzyme, syphilis serology, and borrelia serology) were obtained by venepuncture and sera were aliquoted and stored frozen $\left(-20^{\circ} \mathrm{C}\right)$ for future studies. Chest radiography and fluorescein angiography was performed in all patients. Selected patients (depending on history, character, and activity of their ocular disease as well as the outcome of the laboratory and radiographic screening procedures) underwent special tests and diagnostic procedures. These tests included computed tomography (CT) of chest or brain, gallium-67 whole body scintigraphy, and Mantoux testing. No evidence of systemic or infectious disease was found at entry to the study in any of the patients after uveitis screening. During follow up, patients were asked for new ocular or systemic symptoms and complete ophthalmic examinations were performed at each visit. Screening for sarcoidosis (angiotensin converting enzyme and chest radiography) was repeated regularly. When patients had symptoms of extraocular diseases they were referred to other medical specialists (pulmonologist, rheumatologist, neurologist, dermatologist) for further evaluation.

In all patients with intermediate uveitis the intraocular inflammation was predominantly localised in the vitreous, pars plana, or peripheral retina. At the time of venepuncture the presence of cystoid macular oedema (CMO), vitreous exudates or snowbank formation, papillitis, and periphlebitis (based on fluorescein angiography) were scored. Disease activity was divided into active or smouldering. Active intermediate uveitis was defined as an exacerbation of the disease with marked inflammatory reaction with cells and debris in the vitreous (Hogan and Kimura, grade 2-4). ${ }^{25}$ In smouldering disease there was no exacerbation but a minimal to moderate cellular infiltration within the vitreous was still present.

Most patients with intermediate uveitis were between 20 and $40(n=48,79 \%)$ years of age at the onset of ocular disease, five were younger than 20 , and 18 were older than 40 . Forty three patients were female and 18 were male. Most of the intermediate uveitis patients were not treated or were taking topical corticosteroid treatment at first presentation to one of our clinics. Only five patients were taking systemic immunosuppression. Mean follow up after venepuncture of the intermediate uveitis patients was 4.5 years with a range of 6 months to 15 years.

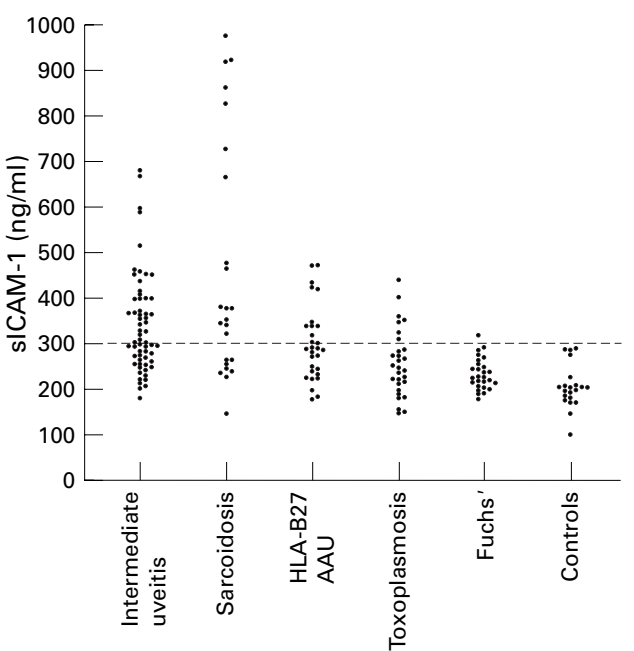

Figure 1 sICAM-1 levels in serum from intermediate uveitis patients, uveitis controls, and normal controls. The line represents the value above which the assay is considered positive. $A A U=$ acute anterior uveitis.

Table 2 sICAM-1 and disease activity in intermediate uveitis

\begin{tabular}{llll}
\hline & $\begin{array}{l}\text { sICAM-1 pos* } \\
(n=34)\end{array}$ & $\begin{array}{l}\text { sICAM-1 neg } \\
(n=27)\end{array}$ & p Value \\
\hline Active & 27 & 12 & $<0.01$ \\
Smouldering & 7 & 15 & \\
\hline
\end{tabular}

* sICAM-1 level above $300 \mathrm{ng} / \mathrm{ml}$.

Statistical analysis was performed using the $\chi^{2}$ test.

Serum samples of 56 uveitis controls with an associated systemic disease and active ocular disease (26 patients with sarcoid anterior uveitis and 30 HLA-B27 positive acute anterior uveitis), 58 uveitis controls without systemic disease (30 toxoplasmic chorioretinitis and 28 Fuchs' heterochromic cyclitis), and 21 healthy controls were collected in the same period as the intermediate uveitis group and stored under similar conditions. In all patients a specific diagnosis of uveitis was made according to the criteria of the International Uveitis Study Group. ${ }^{26}$ Serum levels of sICAM-1 were detected using a commercially available enzyme linked immunosorbent assay (ELISA, human soluble ICAM-1 immunoassay, R\&D Systems) according to the manufacturer's instructions. The concentration of SICAM-1 in samples was calculated by comparing the obtained ELISA reading with a standard curve generated from standards of known concentration supplied with the kit. Adsorbance was determined at $450 \mathrm{~nm}$. A value of $300 \mathrm{ng} / \mathrm{ml}$ was considered as elevated. This value was calculated by taking the mean plus two times the standard deviation of our controls. This value is in accordance to the upper limit for healthy individuals as provided by the manufacturer.

Statistical analysis of the data was performed using Mann-Whitney $\mathrm{U}$ test and $\chi^{2}$ test.

\section{Results}

Elevated sICAM-1 levels (>300 ng/ml) were found in 34 of the 61 patients with intermediate uveitis (56\%) (Table 1, Fig 1). sICAM-1 levels in intermediate uveitis were significantly different when compared with toxoplasmosis, 
Table 3 sICAM-1 and clinical aspects of intermediate uveitis

\begin{tabular}{llcl}
\hline & $\begin{array}{l}\text { Elevated } \\
\text { sICAM-1 } \\
(n=34)\end{array}$ & $\begin{array}{l}\text { Normal } \\
\text { sICAM-1 } \\
(n=27)\end{array}$ & p Value \\
\hline CMO† & 14 & 7 & $\mathrm{~ns}$ \\
Vitreous exudates & 24 & 12 & $<0.05$ \\
Papillitis & 6 & 1 & $\mathrm{~ns}$ \\
Periphlebitis & 8 & 6 & $\mathrm{~ns}$
\end{tabular}

*sICAM-1 level above $300 \mathrm{ng} / \mathrm{ml}$.

†Cystoid macular oedema.

Statistical analysis was performed using the $\chi^{2}$ test.

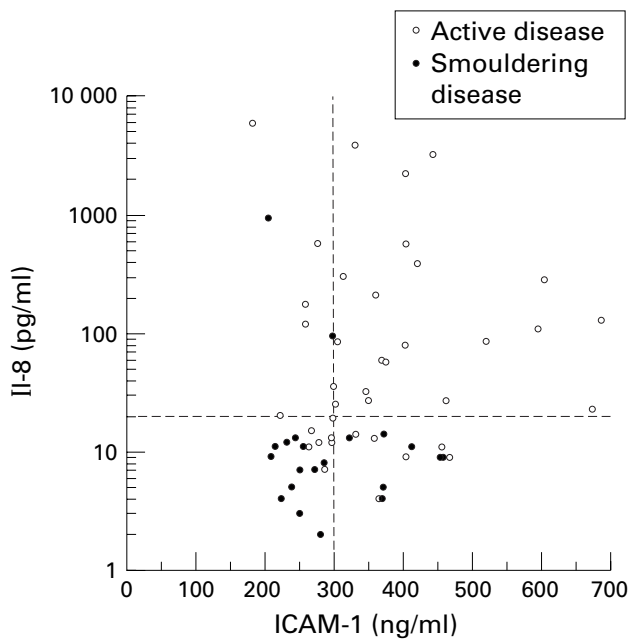

Figure 2 sICAM-1 levels and IL-8 levels in serum from patients with intermediate uveitis. The broken lines represent the value above which the tests are considered positive. The patients with increased sICAM-1 and increased IL-8 had active disease.

Fuchs' heterochromic cyclitis, and normal controls $(p<0.001)$. High levels of sICAM-1 were also seen in 18 of the 26 patients with sarcoidosis $(69 \%)$ and 11 of the 30 HLA-B27 acute anterior uveitis patients $(37 \%)$. Of the intermediate uveitis patients with active ocular disease ( $n=39), 27(70 \%)$ had elevated circulating sICAM-1 levels compared with seven out of 22 patients with smouldering intraocular inflammation $(\mathrm{p}<0.01)$ (Table 2$)$. When analysing the relation between sICAM-1 and ocular characteristics, only vitreous exudates were found more often in patients with elevated sICAM-1 compared with patients with normal sICAM-1 levels $(\mathrm{p}<0.05)$ (Table 3$)$. The presence of CMO, papillitis, and periphlebitis did not significantly differ between patients with elevated and normal sICAM-1 levels. Papillitis was seen in seven patients of whom six had elevated sICAM-1 levels. Owing to the small numbers this finding did however not reach statistical significance. No association was

Table 4 Relation of sICAM-1 levels and IL-8 levels to the presence of systemic disease at the end of the study in patients with intermediate uveitis

\begin{tabular}{lllll}
\hline & $\begin{array}{l}s I C A M-1 \uparrow \\
I L-8 \uparrow(21)\end{array}$ & $\begin{array}{l}s I C A M-1 \uparrow \\
I L-8 n(13)\end{array}$ & $\begin{array}{l}s I C A M-1 n \\
I L-8 \uparrow(8)\end{array}$ & $\begin{array}{l}s I C A M-1 n \\
I L-8 n(19)\end{array}$ \\
\hline Multiple sclerosis & 2 & - & - & 2 \\
Sarcoidosis & 1 & - & - & - \\
Multiple sclerosis suspect & 2 & - & 1 & - \\
Sarcoidosis suspect & 4 & - & - & 1 \\
\hline
\end{tabular}

$\mathrm{n}=$ normal levels; $\uparrow=$ elevated levels.

An established or suspected systemic disease was significantly more found in the 21 patients with elevated sICAM- 1 and IL- 8 compared with the other 40 intermediate uveitis patients $\left(p<0.01, \chi^{2}\right.$ test). observed between serum ICAM-1 levels and age, sex of patients, or systemic corticosteroid treatment of ocular disease.

In our previous study high IL-8 levels $(>20$ $\mathrm{pg} / \mathrm{ml}$ ) were found in 27 of these 61 patients with intermediate uveitis. Raised IL-8 levels were significantly associated with active disease $(\mathrm{p}<0.001)$ and the presence of vitreous exudates $(\mathrm{p}<0.001)$, papillitis, and periphlebitis $(\mathrm{p}<0.01) .{ }^{8}$ Raised sICAM-1 or IL- 8 are found in intermediate uveitis, a finding that remains significant even after correction of the $p$ value for the number of analysis performed.

Analysis of the levels of sICAM-1 and IL-8 in the 61 patients with intermediate uveitis showed that all patients with increased sICAM-1 and increased IL-8 had active disease (Fig 2).

At the end of the study (mean follow up of 4.5 years after blood sampling) a systemic disease was found in five patients and was suspected in eight patients (Table 4). Four patients developed multiple sclerosis; two had high levels of sICAM-1 and IL-8 and both had active ocular disease; the other two patients who developed multiple sclerosis had smouldering ocular disease with low sICAM-1 and IL-8 levels, one of these was on systemic immunosuppression. In one patient a diagnosis of sarcoidosis was made 2 years after the entry into the study; this patient had active disease with high levels of sICAM-1 and IL-8. Three patients developed optic neuritis and other neurological symptoms and were suspected of having multiple sclerosis but have not yet fulfilled the Poser criteria for a definite $\mathrm{MS}^{27}$ : all had active ocular disease. Two of them were sICAM-1 positive and all were IL-8 positive. In five additional patients serum angiotensin converting enzyme levels increased from normal to high levels and/or had chest radiographic or gallium-67 scintigraphic patterns typical of sarcoidosis without, however, confirmation of the diagnosis by histological examination of biopsy specimens. These were suspected of having sarcoidosis; four had active disease with high sICAM-1 and IL-8 levels, one patient treated with systemic steroids had smouldering disease with low sICAM-1 and IL-8 levels. An established or suspected systemic disease was significantly more often found in the 21 patients with intermediate uveitis with increased levels of both sICAM-1 and IL-8 compared with the other 40 patients with intermediate uveitis $(p<0.001)$. No statistical difference was found in frequency of systemic disease when the group of patients with elevated sICAM-1 and elevated IL-8 was compared with patients with elevated SICAM-1 and normal IL-8 levels, with patients with normal sICAM-1 and elevated IL-8 levels, or with the group of intermediate uveitis patients with normal sICAM-1 and normal IL-8 levels.

\section{Discussion}

In this study we found raised levels of sICAM-1 in the serum of $56 \%$ of patients with intermediate uveitis. Increased levels of sICAM-1 were significantly related to intraocular inflammation. Increased levels of 
sICAM-1 were also correlated with the presence of increased IL-8 levels in patients with active intermediate uveitis. These findings suggest that a systemic inflammatory reaction may have a role in patients with intermediate uveitis.

ICAM-1 seems to have a role in a variety of cellular interactions involved in inflammatory and immunological responses. ${ }^{16}$ It is expressed on various cell types, is upregulated by cytokines, and plays an important part in leucocyte transendothelial migration and the cell-cell contact. ${ }^{10-15}$ ICAM-1 was reported to be expressed on endothelial cells of retinal and choroidal blood vessels and the retinal pigment epithelium in uveitic eyes. ${ }^{9}$

A soluble form of ICAM-1 has been detected at increased concentrations in patients with inflammatory and autoimmune diseases such as rheumatoid arthritis, spondylarthropathies, sarcoidosis, and multiple sclerosis. ${ }^{18-23}$ High levels of sICAM-1 correlated with clinical active disease or enhancing lesions on magnetic resonance imaging in patients with multiple sclerosis and were also correlated with CSF pleocytosis, TNF $\alpha$ levels, and blood-brain barrier damage. ${ }^{22} 23$ These findings raise the possibility that circulating ICAM-1 serves as a marker of acute inflammatory events in multiple sclerosis. Increased sICAM-1 levels were also correlated with disease activity in patients with pulmonary sarcoidosis. ${ }^{2021}$

Raised circulating ICAM-1 levels have been reported earlier in intermediate uveitis, sarcoid associated uveitis, and retinal vasculitis. ${ }^{24} 2829$ The first study reported by Arocker-Mettinger et $a l^{28}$ did not detect a relation with intraocular inflammatory activity. Our findings are in agreement with later studies of Palmer et al ${ }^{24}$ and Zaman et $a l^{29}$ who also found a relation between ocular disease activity and sICAM-1 levels. We did not find a relation between sICAM-1 and manifest periphlebitis noted previously in patients with retinal vasculitis, possibly because the number of patients with intermediate uveitis with periphlebitis in our study was quite low.

Furthermore, we found high levels of sICAM-1 in patients with uveitis and other systemic diseases (sarcoid associated uveitis and HLA-B27 positive acute anterior uveitis). This finding may indicate that idiopathic intermediate uveitis is not an isolated eye disease but is probably the first manifestation of an otherwise subclinical systemic disease.

Evidence for a systemic immune response in idiopathic uveitis patients has been found previously. Activated $\mathrm{T}$ cells were found in the peripheral blood of patients with idiopathic uveitis. ${ }^{30}$ Increased levels of sIL-2R were found in patients with retinal vasculitis and systemic inflammatory diseases, ${ }^{31}$ and in patients with Fuchs' heterochromic cyclitis and juvenile chronic arthritis. ${ }^{32}$ These investigators did not find elevated levels of sIL-2R in patients with intermediate uveitis and sIL-2R was therefore not considered to be a useful predictor in intermediate uveitis. ${ }^{32}$
A major problem in the management of intermediate uveitis is still the fact that the currently available laboratory tests cannot predict the course and prognosis of ocular disease nor identify an underlying systemic disease. Therefore the introduction of new serological tests is necessary. ${ }^{33}$ We have already described that increased levels of IL-8 were associated with active ocular disease in patients with intermediate uveitis and that this was not related to an acute phase reaction because $\mathrm{C}$ reactive protein levels were not raised.

At the end of the present study a relation with systemic disease was suspected in 13 $(21 \%)$ of the patients with intermediate uveitis. A definite systemic disease was found in five patients and eight patients had incomplete evidence of associated systemic disease. Nine of these patients were sICAM- 1 and 10 were IL- 8 positive at entry of the study; furthermore all patients who were sICAM-1 positive also had high levels of IL-8. None of the controls was sICAM-1 positive, but five out of 29 controls were positive in the IL- 8 study, which indicates that the measurement of sICAM-1 is probably more specific than the measurement of IL- 8 .

In summary, this study shows that elevated sICAM-1 levels are associated with ocular disease activity and that elevated levels of both sICAM-1 and IL- 8 seem to predispose to a later development of an associated systemic disease in patients with intermediate uveitis.

1 Smith RE, Godfrey WA, Kimura SJ. Chronic cyclitis. I Course and visual prognosis. Trans Am Acad Ophthalmol Otolaryngol 1973;77:760-8.

2 Henderly DE, Genstler AJ, Smith RE, et al. Changing patterns of uveitis. Am F Ophthalmol 1987;103:131-6.

3 Weiner A, Ben Ezra D. Clinical patterns and associated conditions in chronic uveitis. Am f Ophthalmol 1991:112:1519 .

4 Malinowsky SM, Folk JC, Puliodo JS. Pars planitis. Curr Opin Ophthalmol 1994;5:72-82.

5 Zierhut M, Foster CS. Multiple sclerosis, sarcoidosis and other diseases in patients with pars planitis. Dev Ophthalmol

6 Malinowsky SM, Pulido JS, Folk JC. Long-term visual outcome and complications associated with pars planitis. Ophthalmology 1993;100:818-25.

7 Boskovich SA, Lowder CY, Meisler DM, et al. Systemic diseases associated with intermediate uveitis. Cleveland Clin $\mathcal{F}$ Med 1993;60:460-5.

8 Klok AM, Luyendijk L, Zaal MJW, et al. Elevated serum IL-8 levels are associated with disease activity in idiopathic intermediate uveitis. Br F Ophthalmol 1998:82:871-4.

9 Whitcup SM, Chan CC, Li Q, et al. Expression of cell adhesion molecules in posterior uveitis. Arch Ophthalmol 1992;110:662-6.

10 Rothlein R, Dustin ML, Marlin SD, et al. A human intercellular adhesion molecule (ICAM-1) distinct from LFA-1. f Immunol 1986;137:1270-4.

11 Marlin SD, Springer TA. Purified intercellular adhesion molecule-1 (ICAM-1) is a ligand for lymphocyte-function associated antigen 1 (LFA1). Cell 1987;51:813-9.

12 Staunton DE, Marlin SD, Stratpwa C, et al. Primary structure of ICAM-1 demonstrates interaction between members of the imminoglobulin and integrin supergene bers of the imminoglobulin

13 Dustin ML, Rothlein R, Bhan AK, et al. Induction by IL1 and interferon-gamma: tissue distibution, biochemistry, and function of a natural adherence molecule (ICAM-1). $\mathcal{F}$ Immunol 1986;137:245-54

14 Rothlein R, Czajkowski M, O'Neill MM, et al. Induction of intercellular adhesion molecule 1 on primary and continuous cell lines by pro-inflammatory cytokines. F Immunol 1988;141:1665-9.

15 Van de Stolpe A, van der Saag PT. Intercellular adhesion molecule-1. 7 Mol Med 1996;74:13-33.

16 Seth R, Raymond FD, Makgoba MW. Circulating ICAM-1 soforms: diagnostic prospects for inflammatory and immune disorders. Lancet 1991;338:83-4.

17 Rothlein R, Mainolfi EA, Czakowski M, et al. A form of circulating ICAM-1 in human serum. F Immunol 1991;147: culating I I

18 Cush JJ, Rothlein R, Lindsey HB, et al. Increased levels of circulating intercellular adhesion molecule 1 in the sera of patients with rheumatoid arthritis. Arthritis Rheum 1993; 36:10098-1102. 
19 Wendling D, Racadot E, Auge B, et al. Soluble intercellular adhesion molecule 1 in spondylarthropathies. Clin Rheumaadhesion molecule 1 in

20 Baumer I, Zissel G, Schlaak M, et al. Shed soluble ICAM-1 molecules in bronchoalveolar lavage cell supernatants and serum of patients with pulmonary sarcoidosis. Lung 1997; 75:105-16

21 Shijubo N, Imai K, Shigehara K, et al. Circulating soluble intercellular adhesion molecule-1 (sICAM-1) in patients with sarcoidosis. Clin Exp Immunol 1996;106:549-54.

22 Hartung HP, Michels M, Reiners K, et al. Soluble ICAM-1 serum levels in multiple sclerosis and viral encephalitis. Neurology 1993;43:2331-5.

23 Sharief MK, Noori MA, Ciardi M, et al. Increased levels of circulating ICAM-1 in serum and cerebrospinal fluid of patients with active multiple sclerosis. Correlation with TNF- $\alpha$ and blood-brain barrier damage. I Neuroimmunol 1993;43:15-22.

24 Palmer HE, Zaman AG, Ellis BA, et al. Longitudinal analysis of soluble intercellular adhesion molecule 1 in retinal sis of soluble intercellular adhesion molecule 1 in

25 Nussenblatt RB, Whitcup SM, Palestine AJ. Uveitis: fundamentals and clinical practice. 2nd ed. Chapter 20. Intermediate uveitis. St Louis: Mosby, 1996:279-88.
26 Bloch- Micel E, Nussenblatt RB. International Uveitis Study Group recommendations for the evaluation of
intraocular inflammatory disaese. Am $\mathcal{F}$ Ophthalmol 1987; 103:234-5.

27 Poser CM, Paty DW, Scheinberg L, et al. New diagnostic criteria for multiple sclerosis:Guidelines for research protocols. Ann Neurol 1983;13:227-30.

28 Arocker-Mettinger E, Steurer-Georgiew L, Steurer M, et al. Circulating ICAM-1 levels in serum of uveitis patients. Curr Eye Res 1992;11 (suppl): 161-6.

29 Zaman AG, Edelsten C, Stanford R, et al. Soluble intercellular adhesion molecule-1 (sICAM-1) as a marker of disease relapse in idiopathic uveoretinitis. Clin Exp Immunol 1994;95:60-5

30 Deschênes J, Char DE, Kaleta S. Activated T lymphocytes in uveitis. Br 7 Ophthalmol 1988;72:83-7.

31 Murray PI, Young DW. Soluble interleukin-2 receptors in retinal vasculitis. Curr Eye Res 1992;11 (suppl): 193-5.

32 Arocker-Mettinger E, Asenbauer T, Ulbrich S, et al. Serum interleukin 2-receptor levels in uveitis. Curr Eye Res 1990;9 (suppl):25-9.

33 Dick AD. Serological tests for monitoring and predicting disease severity, course, and outcome of autoimmune intraocular inflammation. Br f Ophthalmol 1998;82:856-7. 